Received: 2013.01.25 Accepted: 2013.04.03 Published: 2013.04.19

Authors' Contribution: Study Design A Data Collection B Statistical Analysis C Data Interpretation D Manuscript Preparation E Literature Search F Funds Collection G

\section{A rare cause of hypocalcemia}

Corresponding Author:

Background:

Case Report:

Conclusions:

Key words:

Full-text PDF:
1 Department of Medicine, University of Florida, Gainesville, FL, U.S.A.

2 Department of Medicine, Hamad Medical Corporation, Doha, Qatar
ABEF 2 Timi Rotimi Omokehinde 


\section{Background}

Hypocalcemia is a disorder of mineral metabolism that occurs when PTH is insufficient to act on end organs (kidney, bone, intestine) -hypoparathyroidism, or end organs irresponsiveness in setting of sufficient PTH to normalize serum calcium - PTH resistance. In the setting of adequately functioning parathyroid glands, and responsive end organs, other causes of hypocalcemia, such as vitamin D deficiency, are characterized by high PTH (secondary hyperparathyroidism). Thus, it is may be useful to broadly characterize hypocalcemia as associated with low PTH or high PTH [1]. One of the causes of high PTH associated hypocalcemia is PTH resistance which is often associated with hypocalcemia, hyperphosphatemia.

\section{Case Report}

A 40 year old gentleman with no prior medical illness presented to the emergency department with 1 day history of generalized malaise and subjective fever. He denies any history of prior similar symptoms. His past medical and surgical history were unremarkable. His social history is significant for being a carpenter, marriage and two children. His family history was unremarkable for chronic illnesses or diseases. The review of systems was otherwise negative. On examination, the patient looks well and not in distress. BP: 150/82, Temperature: $37.4^{\circ} \mathrm{C}$, Pulse: $100 \mathrm{bpm}$, Respiratory rate: $18 / \mathrm{min}, 02$ sat: $97 \%$ on room air, weight: 62 kg, height: $168 \mathrm{~cm}, \mathrm{BMI}: 22$, General physical examination was unremarkable. Sample of laboratory investigations were as follows: Corrected Calcium 1.37 $\mathrm{mmol} / \mathrm{L}$ (2.1-2.6), Ionized Calcium 1.05 (1.18-1.32 mmol/L), Phosphorous $1.72 \mathrm{mmol} / \mathrm{L}$ (0.87-1.45), Magnesium 0.72 $\mathrm{mmol} / \mathrm{L}$ (0.65-1.05), BUN $3.4 \mathrm{mmol} / \mathrm{L}$ (1.7-8.3), Creatinine 86 umol/L (62-124), Bicarbonate $26 \mathrm{mmol} / \mathrm{L}$ (24-30), Albumin 44 g/L (35-50), Alkaline Phosphatase 114 U/L (40-129), Vitamin D 30 ng/ml (30-80), Parathyroid hormone (immunoreactive) $114 \mathrm{pg} / \mathrm{ml}$ (15-65), Urine Calcium 0.2, Urinary Calcium Creatinine ratio 0.02 (>0.4). Other biochemical analysis including complete blood count, thyroid stimulating hormone, free thyroxine and cortisol levels were all normal. EKG showed normal sinus rhythm with normal Q-T interval. CXR was within normal.

The patient was managed with both parenteral and oral Calcium supplements. The corrected serum calcium reached a plateau of $2.07 \mathrm{mmol} / \mathrm{L}$ and phosphorus reached a nadir of $1.42 \mathrm{mmol} / \mathrm{L}$. iPTH was to be repeated following institution of therapy at follow up in out-patient clinic. At the time of this write up, he was discharged to home on oral calcium carbonate tablets with an appointment for follow up in the clinic.

\section{Discussion}

Approximately, $500 \mathrm{mg}$ of calcium is removed from the bones daily and replaced by an equal amount. Normally, the amount of calcium absorbed by the intestines is matched by urinary calcium excretion in order to maintain homeostasis. Despite these enormous fluxes of calcium, the levels of ionized calcium remain stable because of the tight control maintained by parathyroid hormone (PTH), vitamin D, and calcitonin through complex feedback loops. These hormones act primarily at the level of bone, kidney, and gastrointestinal tract. Calcium levels are also affected by magnesium and phosphorus [2]. The parathyroid gland has a remarkable sensitivity to ionized serum calcium changes. These changes are recognized by the calcium-sensing receptor (CaSR) and a slight decrease in serum ionized calcium stimulates the chief cells of the parathyroid gland to secrete PTH [1].

The most common causes of hypocalcemia with accompanied high PTH are hypoalbuminemia, hypomagnesemia, hyperphosphatemia, and PTH resistance. Calcium is transported in blood partly bound to protein (roughly $45-55 \%$ ), mostly serum albumin. The total serum calcium may be reduced as a result of hypoalbuminemia, but concentration of hormonally active, free ionized calcium remains unchanged [3]. Hypomagnesemia may also cause hypocalcemia due to PTH resistance, hence the high PTH. Hyperphosphatemia associated hpocalcemia is primarily due to bone deposition and extraskeletal tissue calcification. Other etiologies include calcium chelation (with citrate, foscanet, lactate for instance) which reduces ionized calcium, but not total serum calcium [4], medication effects such as reduced oteoclastic bone resorption in the setting of bisphosphonates and acute inhibition of PTH release with calcimimetic agent, notably cinacalcet $[5,6]$.

On the other hand, hypocalcemia with low PTH is often encountered following parathyroid gland destruction post surgery and in the rare cases of acquired and/or familial autoimmune disorders (such as in polyglandular autoimmune disorder type 1.) Infiltrative diseases such as hemochromatosis, granulomas can also present with hypocalcemia and low PTH [7]. Isolated or familial cases of activating mutation of CaSR may also present in similar manner where PTH is not released at serum calcium concentration that normally stimulate PTH production from the glands.

Our patient had a normal level of serum albumin, magnesium, vitamin D, kidney function, and normal renal calcium excretion (ruling out the possibility of renal tubular loss). Along with the constellation of hypocalcemia, hyperphosphotemia, and high parathyroid hormone levels, he was diagnosed with parathyroid hormone resistance - pseudohypoparathyroidism.

Pseudohypoparathyroidism is characterized by end-organ resistance to the effects of PTH. PTH binds to the PTH receptor, which, activates CAMP through guanine nucleotide regulatory 
G-protein for an effective signal transduction. PTH resistance is encountered as a loss-of-function mutation in the gene that encode the alpha subunit of the G-protein that is coupled to the PTH receptor to activate the adenyl cyclase required for signal transduction that produces end-organ response to PTH. There is a failure of signal transduction, hence the PTH resistance [8]. Pseudohypoparathyroidism is classified into types I and II. Type I is further subdivided into la, Ib, and Ic. Type la comprises the biochemical features of pseudohypoparathyroidism, blunted urinary CAMP response to exogenous PTH administration along with the somatic features of Albright Hereditary Osteodystrophy (AHO), such as short stature, obesity, developmental delay, subcutaneous calcification, short fourth metacarpal bone, and round face. The mode of inheritance is autosomal dominant pattern with gene imprinting in which only female can transmit the full disease. Type la is unlikely in our patient who presented in adulthood without AHO's characteristic features [9].

Type Ib pseudohypoparathyroidism harbors autosomal dominant inherent pattern with familial distribution but without somatic features of AHO. In most patients with PHP-Ib, global G-proteins mutations have not been found, hence the absence of AHO features. The hormonal resistance is usually limited to the PTH-dependent actions in proximal renal tubules in which G-protein is paternally imprinting and consequently the impaired urinary CAMP response to PTH and other biochemistries abnormalities [8].

\section{References:}

1. Richardi D, Brown EM: Physiology and pathophysiology of the calcium-sensing receptor in the kidney. Am J Physiol Renal Physiol, 2010; 298: F485-99

2. Sarko J: Bone and mineral metabolism. Emerg Med Clin North Am, 2005; 23(3): 703-21, viii

3. Heiko L, Henning K et al: Preconditions of Hemostasis in Trauma: A Review. The Influence of Acidosis, Hypocalcemia, Anemia and Hypothermia on Functional Hemostasis in Trauma. J Trauma, 2010; 65(4): 951-60

4. Jacobson MA, Gambertoglio JG et al: Foscanet-induced hypocalcemia and effects of foscanet on calcium metabolism. J Clin Endocrinol Metab, 1991; 72: $1130-35$
Patient with type Ic pseudohypoparathyroidism presents with resistance to multiple hormonal receptors. The patients are phenotypically similar to type la but with normal functioning G-protein.

In type /I pseudohypoparathyroidism, PTH raises CAMP normally but fails to increase levels of serum calcium or urinary phosphate excretion, suggesting that the defect is located downstream of the generation of cAMP [9]. There is usually no familial pattern (and sporadic if present) as seen with patients in all Type I classification. These patients present with hypocalcemia, hypophosphaturia, and elevated immunoreactive PTH (iPTH) levels which are also seen in vitamin D deficiency. These abnormal lab values often return to normal upon adequate vitamin $\mathrm{D}$ supplementation.

\section{Conclusions}

Our patient was classified as Type II. He presented noncontributory familial history and absence of phonotypical features of AHO. Type II is considered the rarest of the classification likely due to its late presentation in adulthood, lack or inconsistent familial distribution, and absence of AHO features. These patients are usually diagnosed incidentally as in our patient and often have benign course.

5. Peter R, Mishra V, Fraser WD: Severe hypocalcemia after being given IV bisphosphonate. BMJ, 2004; 328(7435): 335-36

6. Chonchol M, Locatelli F et al: A Randomized, Double-Blind, Placebo-Controlled Study to Assess the Efficacy and Safety of Cinacalcet HCL in Participants with CKD Not Receiving Dialysis. Am J Kidney Dis, 2009; 53(2): 197-207

7. Carpenter TO, Carnes DL Jr et al: Hypoparathyroidism in Wilson's disease. N Engl J Med, 1983; 308: 873-77

8. Elena L, Murat B et al: Phenotypic and Molecular Genetic Aspects of Pseudohypoparathyroidism Type Ib in a Greek Kindred: Evidence for Enhanced Uric Acid Excretion Due to Parathyroid Hormone Resistance. J Clin Endocrinol Metab, 2004; 89(12): 5942-47

9. Giovana M: Pseudohypoparathyroidism: Diagnosis and Treatment. J Clin Endocrinol Metab 2011; 96(10): 3020-30 